\title{
The prevalence and socio-behavioural and clinical covariates of oral health related quality of life in Ugandan mothers with and without HIV-1
}

Nancy Birungi ${ }^{*}$ (D), Lars Thore Fadnes ${ }^{2,3}$, Ingunn Marie Stadskleiv Engebretsen ${ }^{4}$, James Kashugyera Tumwine ${ }^{5}$ and Anne Nordrehaug Åstrøm ${ }^{1}$ ANRS 12174 and 12341 study groups

\begin{abstract}
Background: There is limited evidence regarding oral health related quality of life of HIV positive populations in sub-Saharan Africa. Focusing HIV positive- and HIV negative Ugandan mothers, this study assessed the influence of HIV status on oral health related quality of life in terms of oral impacts on daily performances, whilst adjusting for clinical- and socio-behavioural factors. We also examined whether any association of clinical and socio-behavioural factors with oral impacts on daily performances vary according to mothers' HIV status.
\end{abstract}

Methods: This cross-sectional study used data from a trial $(n=164)$ and a comparison group $(n=181)$. The trial comprised of mothers with HIV-1 participating in the ANRS 121741-PROMISE-PEP-trial (NCT00640263) conducted between 2009 and 2013 and from the ANRS 12341-PROMISE-PEP-M\&S follow-up study conducted in 2017. The comparison group comprised of HIV negative mothers recruited in 2017. Interviews and clinical oral examinations were performed. The oral health related quality of life was assessed using the oral impacts on daily performances frequency scale. Caries experience and gingival bleeding were assessed using the World Health Organization's Decayed, Missed and Filled teeth indices and community periodontal index. Logistic and negative binomial regression analyses were performed.

Results: $29 \%$ of HIV-1 positive and 32\% among the comparison reported any oral impact on daily performance. In adjusted logistic regression analysis, HIV status was not significantly associated with oral impacts on daily performances. Mother's self-reported oral health, caries experience, gingival bleeding and oral health related quality of life of their children were independently associated with oral impacts on daily performances. Corresponding prevalence ratios and 95\% confidence intervals were: 0.3 (0.2-0.6), 1.8 (1.0-3.2), 1.1 (1.0-1.1), and 2.1 (1.1-4.3). No significant interaction between HIV status and covariates were observed.

Conclusions: Oral health related quality of life was substantially impaired in Ugandan mothers but did not discriminate between HIV positive and negative participants. Mothers with impaired oral health related quality of life were more likely to have dental caries and children with impaired oral health related quality of life. HIV positive and negative mothers in Uganda deserve special attention regarding their oral disease and quality of life status.

Keywords: Dental caries, Oral health, Quality of life, HIV, Oral impacts on daily performances

\footnotetext{
*Correspondence: Nancy.Birungi@uib.no

${ }^{1}$ Department of Clinical Dentistry, University of Bergen, Bergen, Norway

Full list of author information is available at the end of the article
} permits use, sharing, adaptation, distribution and reproduction in any medium or format, as long as you give appropriate credit to the original author(s) and the source, provide a link to the Creative Commons licence, and indicate if changes were made. The images or other third party material in this article are included in the article's Creative Commons licence, unless indicated otherwise in a credit line to the material. If material is not included in the article's Creative Commons licence and your intended use is not permitted by statutory regulation or exceeds the permitted use, you will need to obtain permission directly from the copyright holder. To view a copy of this licence, visit http://creativecommons.org/licenses/by/4.0/. The Creative Commons Public Domain Dedication waiver (http://creativeco mmons.org/publicdomain/zero/1.0/) applies to the data made available in this article, unless otherwise stated in a credit line to the data. 


\section{Introduction}

The global availability and scale-up of antiretroviral treatment (ART) over the last 20 years has increased the life expectancy of persons living with the human immune virus (PLHIV) [1]. Thus, focus of treatment has shifted from opportunistic infections caused by a failing immune system to managing chronic non-communicable diseases that could occur in one's lifetime. Globally, there are 38 million PLHIV and of them 20.7 million live in eastern and southern Africa [1]. With further scale up of the access to ART the former and later figures are expected to grow.

According to the latest global burden of disease studies (GBD), dental caries and periodontitis are non-communicable diseases, recognized to be among the leading causes of disabilities in many countries [2]. Thus, about 3.5 billion people worldwide live with dental diseases whereas $35 \%$ ( 2.5 billion) present with untreated caries in the permanent dentition. Moreover, severe periodontitis has been reported to be the sixth -most prevalent condition affecting $10.8 \%$ (743 million) of the global population. Corresponding figure for tooth loss is $2.3 \%$ (158 million).

There has been a growing recognition of subjective health indicators, such as the health-related quality of life (HRQoL) and oral health related quality of life [35]. Oral health related quality of life (OHRQoL) measures or socio-dental indicators have proved to be valid instruments to assess oral health as well as the quality of care or services of populations or groups of people [4]. Unlike clinical indicators, OHRQOL measures are subjective and capture multiple dimensions and consequences of oral disease. The dimensions are not limited to the physical consequences of disease but extend to emotional, social, financial and psychological aspects as well. A frequently used socio-dental indicator is the oral health impact on daily performances (OIDP) instrument; developed to assess oral impacts that seriously affect a person's daily life activities [6]. The OIDP assesses outcomes of disability, handicap aspects and social disadvantages of oral conditions which are part of the broader World Health Organisation (WHO)'s International Classification of Impairments, Disabilities and Handicaps (ICIDH) [7]. The ICIDH enables empirical exploration of links between different dimensions of the following key concepts, impairments, functional limitations, pain and discomfort, disability, and handicap. Locker et al. [8] amended ICIDH for dentistry. The OIDP Scale consists of 8 items that cover physical, psychological, and social dimensions of daily living. The frequency OIDP scale has demonstrated acceptable psychometric properties in the context of an oral health survey among Ugandan adolescents. Thus, discriminant and construct validity were demonstrated in that the OIDP scores varied systematically in the expected direction with missing teeth and self-report indicators of oral health status, respectively [9]. This scale has also been used to assess the impact on oral health related quality of life of periodontal disease focusing pregnant women in Uganda [10].Oral health related quality of life has been assessed among people living with HIV [11]. Australian studies, conducted before and after the implementation of the mass scaleup of ART, have reported more social impacts of oral diseases in HIV positive adults compared to HIV negative peers [12, 13]. Accordingly, a US study revealed that women with HIV had on average $10 \%$ poorer OHRQoL than uninfected women in a control group when adjusting for confounding behavioural- and clinical factors [14]. In addition, a study from Portugal reported that HIV status had a negative impact on OHRQOL after adjustment of the number of decayed teeth, prosthodontic treatment needs and drug use [15]. Tomar et al. [16] identified socio-demographic covariates of OHRQoL in terms of sex, race/ethnicity, living situation (such as prison or other institutional settings) and smoking status among patients with HIV receiving primary care in South Florida. Similar findings are reported in a recent study of US women [17]. In two Brazilian and one Malaysian study, OHRQOL of PLHIV were independently associated with general HRQOL) [18, 19]. Another Brazilian study revealed that functional and psychosocial oral impacts were weakly correlated with general quality of life measures and concluded that the functional and psychosocial oral impacts and the general HRQOL scores represented different constructs [20]. It is also evident that parents self-perceived- and objective oral health is associated with that of their offspring [21-23]. However, the association of parent's quality of life with children's quality of life is not yet established.

Studies considering OHRQoL among adults living with HIV have most frequently been conducted in high-and middle-income countries without inclusion of a HIV negative control group [11, 13, 15, 17, 20, 24-26]. Few studies have evaluated impacts of HIV status on OHRQoL in the context of clinical and socio-behavioural factors of adult populations living in sub-Saharan African cultural contexts. Given the huge burden of HIV in Sub Saharan Africa and the increased life expectancy of PLHIV, information regarding OHRQOL may inform a holistic management of PWHIV.

Focusing HIV positive- and HIV negative Ugandan mothers, this study assessed the influence of HIV status on OHRQoL in terms of oral impacts on daily performances, OIDP, whilst adjusting for clinical- and sociobehavioural factors. We also examined whether any 
association of clinical and socio-behavioural factors with OIDP vary according to mothers' HIV status.

\section{Methods}

This cross-sectional study compares participants from a trial cohort- with a comparison group. The trial cohort included women with HIV-1 residing in the Ugandan site (Mbale, Eastern Uganda) of the multi-center efficacy trial ANRS12174 PROMISE- PEP trial (ClinicalTrials.gov, number NCT00640263). In (that) trial, HIV-1 peri-exposure prophylaxis with ritonavir boosted lopinavir- (LPV/r) or Lamivudine (3TC), respectively, were given to the infants of the mothers who were HIV-1 positive at recruitment. The mothers got standard care and no intervention themselves. All mothers with HIV were diagnosed with the subtype HIV-1. The trial conducted between 2009 and 2013, included pregnant women with HIV-1, recruited at gestational age of 28-40 weeks at antenatal clinics in four African sites. Inclusion criteria for mother was age 18 years or older, intention to continue breastfeeding, and being HIV-1 positive, and not being eligible for ART [either clinically or because CD4 (cluster of differentiation 4) count $>350$ cells $/ \mu \mathrm{L}$ at that time)]. All eligible mothers and infants followed the routine prevention of mother to child (PMTCT) with antepartum zidovudine (ZDV), intrapartum nevirapine (NVP), zidovudine-lamivudine (ZDV/3TC) for mothers and NVP for infants 7 days postpartum. The ANRS12174 PROMISE- PEP trial is described in detail in a previous paper [27].

In 2017, 244 out of 278 mothers with HIV-1 infection and their HIV exposed uninfected children, HEU, were eligible for re-enrolment in the follow-up study: the PROMISE-PEP Mechanism Safety study (PROMISE-PEP M\&S ANRS12341). A total of $67 \%$ of the eligible cohort of HIV-1 positive mothers (164/244) and their HEU children were followed-up leaving $32 \%(n=112)$ lost to follow-up.

For the comparison group, 199 HIV unexposed uninfected (HUU) children, matched on age and sex with 164 HEU and their HIV negative mothers were recruited into the study. They were recruited purposively from communities located in Mbale, Eastern Uganda, which was the site for the ANRS12174 PROMISE- PEP trial. Of the 199 HUU mothers- in the comparison group, 19 were excluded due to a positive HIV-1 test result, leaving 181 HIV negative mothers enrolled. Mothers in the comparison group were tested for their HIV-1 status using serial and parallel HIV rapid testing with Determine, Stat-Pak and Uni-Gold, three test algorithms as recommended by the Ugandan Ministry of Health [28].

Thus, the present study is based on information from interviews and clinical oral examinations of 164 HIV-1 positive-mothers from the trial cohort and their 181 HIV-1 negative controls recruited into the follow up study in 2017 (mothers participating in the follow-up study from the trail cohort and 2017 recruitment of the comparison group, respectively). Mothers from these two groups are hereafter described as HIV-positive and HIVnegative mothers.

\section{Interviews with HIV-1 positive mothers and HIV - 1 negative controls}

Two trained interviewers performed face-to-face interviews with mothers using semi-structured interviews in one of the local Ugandan languages, Lumasaaba. The interview was constructed in English and translated into Lumasaba for use in the field. The instruments had been reviewed previously by project staff for semantic, experiental and conceptual equivalence of the source version. Sensitivity to culture and selection of appropriate words were considered [27, 29]. Mothers responded to questions about themselves. Information was documented on case record forms (CRFs) and electronically with Capture software System (Clinsight) and Epidata program www. epidata.dk for the clinical oral examinations.

Socio-demographic characteristics were assessed in terms of level of education, and age. Level of education was categorized into 'did not finish primary school (1), end of primary school (2) higher education' (3). Mothers' perceived health was assessed as very poor (1), poor (2), fair (3), good (4), very good (5). Mothers' perceived treatment need had the following response categories (1) not at all, (2) very little (3) to some extent, (4) considerably, (5) a great deal and recategorized to not at all/very little (0), to some extent/considerably/a great deal (1). The OHRQOL of mothers' children was assessed using the Early Childhood Oral Health Impact Scale (ECOHIS) previously tested for its psychometric properties in the context of Ugandan pre-school children [30].

The main outcome variable, oral health-related quality of life of HIV -1 positive mothers and their negative controls was assessed using the Ugandan version of the eight-item OIDP scale previously shown to have acceptable psychometric properties among pregnant women in Uganda [10]. The OIDP questions assessed whether in the previous 6 months the respondents had problems with the following items; eating, speaking, cleaning teeth, smiling, sleeping, work performance, social contact and emotional state. For ease of interpretation in this study population, a dichotomous response scale was used in terms of no (0) and yes (1). Additive count scores, OIDP extent, were created from 8 dummy (0-8) OIDP variables, indicating number of daily activities affected. Subsequently, the OIDP extent scores (0-8) were dichotomised, yielding the categories (0) no oral impact/no 
daily performances affected and (1) affected on at least one daily performance/ at least one oral impact [31]. The dichotomized OIDP extent score was used in the crosstabulation and logistic regression analyses.

\section{Clinical oral examination}

Two experienced and calibrated dental surgeons (NB and MM) performed oral assessments among the study participants and duplicated full-mouth oral clinical examination among 26 women not included in the main study for reliability measurements. Dental caries was assessed on surface and tooth level (5 surfaces per tooth) in terms of decayed (D), missing (M), and filled (F) surface/teeth (DMFS/DMFT) in accordance with the World Health Organization (WHO) guidelines for field conditions [32]. Each surface was recoded 0 for sound and 1 for caries experience and documented as decayed if it was visually cavitated with the aid of a dental mirror and periodontal probe. A surface was recorded filled when treated and a tooth was recorded missing when extracted due to caries, as confirmed by the participant. To assess gingival bleeding of the individual, the modified community periodontal index (CPI) was used [32]. Each tooth was scored according to the presence or absence of gingival bleeding, using a periodontal probe across the gingival margins of the teeth. An individual score of 'presence of gingival bleeding' was given if bleeding on probing was scored for at least one tooth in the mouth.

\section{Statistical analysis}

STATA SE 16 (College Station, Texas 77845 USA) was used for data analysis. Cross-tabulation and Chi-square tests were used with categorical variables to assess the crude associations of socio-demographic- and clinical factors and OIDP items according to HIV-1 status. The OIDP scale was used as a count (OIDP range 0-8) as well as a dichotomized outcome variable (OIDP $=0$, OIDP $>0$ ). Socio-behavioural and clinical covariates statistically significantly associated both with HIV-1 status and OIDP in unadjusted analyses were included in the logistic- and negative binomial regression models as potential confounding variables. Logistic regression was used with the OIDP as a binary outcome measure reporting prevalence ratio and 95\% Confidence intervals (PR, 95\% CI). Negative binomial regression was applied with OIDP as a count variable because of its skewed distribution reporting incidence rate ratios and $95 \%$ confidence intervals (IRR, 95\% CI). P-values less than 0.05 were considered statistically significant. The intra examiner agreement and internal consistency of the OIDP scale were assessed using Cohen's Kappa and Cronbach's alpha, respectively. Pairwise interactions between socio-demographic and clinical factors and HIV status upon OIDP were included if the significance criterion set at 0.05 and 0.1 was met.

\section{Results}

A total of 345 mothers (164 HIV positive and 181 confirmed HIV negative) completed interviews and underwent a full-mouth clinical oral examination. Cronbach's alpha for the 8 OIDP items was 0.91. (0.90 in the HIV positive and 0.92 in HIV negative). Cronbach's alpha of the total ECOHIS scale was 0.93. The calibration exercise comparing DMFT scores within and between examiners, revealed Cohen's Kappa values for intra- and inter-examiner reliability of median Kappa (interquartile range) 0.7 $(0.5-0.9)$ and $0.6(0.4-0.8)$, respectively.

Table 1 show that age, type of income, self-reported oral health status, presence of gingival bleeding, caries experience, oral health related information received and child/family oral impacts according to the ECOHIS scale differed statistically significantly between HIV positive and negative mothers. The median age (25-75 percentiles) in those with and without HIV was 35 (30-40) and 31 (25-38) Above half of the participating mother were $18-32$ years; $42 \%$ and $38 \%$ of respectively, HIV positive and negative mothers reported higher education. Corresponding figures regarding need for dental treatment were $13 \%$ and $13 \%$, respectively. A higher prevalence of HIV negative than HIV positive mothers reported good oral health status (67\% versus $56 \%$ ) and caries experience $(\mathrm{DMFT}>0)(81 \%$ versus $71 \%)$. Prevalence of confirmed child/family impacts according to the ECOHIS scale (ECOHIS $>0$ ) were highest among HIV negative mothers, whereas a higher prevalence of HIV positive than HIV negative mothers confirmed having received oral health related information.

As shown in Table 2, difficulty in eating and enjoying contact with people were respectively, the most and least frequently reported impacts among both HIV positive- and negative mothers. Totals of $32.2 \%$ negative and 29.2\% positive mothers reported at least one oral impact on daily performance (OIDP $>0$ ). Among HIV positive mothers, all oral impacts discriminated statistically significantly between those reporting good and poor oral health. Among HIV negative mothers, three impacts (difficult eating, difficult speaking, difficult cleaning teeth) discriminated significantly according to their selfreported oral health status.

As shown in Table 3, unadjusted analysis of mother's socio-behavioural- and clinical characteristics by OIDP status revealed statistically significant associations for perceived oral health, presence of gingival bleeding, mother's caries experience and total ECOHIS scores. When covariates significantly associated with both HIV status and OIDP were included as potential confounding 
Table 1 Mothers' socio-demographic-and self-reported oral health characteristics by HIV status. Percentages of those being HIV negative- and positive

\begin{tabular}{|c|c|c|c|}
\hline & HIV negative & HIV positive & Total \\
\hline & $\%(n)$ & $\%(n)$ & $\%(n)$ \\
\hline $\begin{array}{l}\text { Median age, years ( } 25-75 \text { percentiles) } \\
\text { Age categories }\end{array}$ & $31(25-38)$ & $31(30-40)$ & $31(28-38)$ \\
\hline $18-32$ years & $57.8(104)$ & $39.2(58)$ & $49.4(162)$ \\
\hline $33+$ years & $42.2(76)$ & $60.8(90) * *$ & $50.6(166)$ \\
\hline \multicolumn{4}{|l|}{ Education } \\
\hline End of primary & $61.8(102)$ & $58.2(82)$ & $60.1(184)$ \\
\hline Higher than primary & $38.2(63)$ & $41.8(59)$ & $39.9(122)$ \\
\hline \multicolumn{4}{|l|}{ Perceived oral health } \\
\hline Poor (very poor, poor, fair) & $33.3(60)$ & $66.7(120)$ & $37.8(129)$ \\
\hline Good (Good, very good) & $66.7(120)$ & $57.1(92) *$ & $67.2(212)$ \\
\hline \multicolumn{4}{|l|}{ Need for dental treatment } \\
\hline No & $86.7(156)$ & $87.2(143)$ & $23.8(71)$ \\
\hline Yes & $13.3(24)$ & $12.8(21)$ & $75.6(34)$ \\
\hline \multicolumn{4}{|l|}{ Presence gingival bleeding } \\
\hline No & $56.4(102)$ & $35.4(58)$ & $46.4(160)$ \\
\hline Yes & $43.6(79)$ & $64.6(106)^{* * *}$ & $53.6(185)$ \\
\hline \multicolumn{4}{|l|}{ Caries experience } \\
\hline No & $28.7(52)$ & $58.3(95)$ & $24.1(83)$ \\
\hline Yes & $71.3(129)$ & $81.1(133)^{*}$ & $75.9(262)$ \\
\hline \multicolumn{4}{|l|}{ ECOHIS -total } \\
\hline No impact & 78.5 (139) & $87.7(142)$ & $82.9(281)$ \\
\hline At least one impact & $21.5(38)$ & $12.3(20) *$ & $17.1(58)$ \\
\hline \multicolumn{4}{|l|}{ Information teeth received } \\
\hline No & $73.2(131)$ & $58.3(95)$ & $66.1(226)$ \\
\hline Yes & $26.8(48)$ & $41.7(68) * *$ & $33.9(116)$ \\
\hline
\end{tabular}

IQR interquartile range, ${ }^{*} p<0.1,{ }^{* *} p<0.05,{ }^{* * *} p<0.001$

Table 2 OIDP items by global measures of oral health in HIV positive and negative mothers. Percentage of mothers with impacts. Criterion validity

\begin{tabular}{|c|c|c|c|c|c|c|c|}
\hline & \multicolumn{3}{|l|}{ HIV POSITIVE } & \multicolumn{4}{|l|}{ HIV NEGATIVE } \\
\hline & $\begin{array}{l}\text { Poor oral health } \\
\%(n)\end{array}$ & Good oral health \% (n) & $\begin{array}{l}\text { Sub-Total } \\
\%(n)\end{array}$ & Poor oral health \% (n) & $\begin{array}{l}\text { Good oral health } \\
\%(n)\end{array}$ & Sub-total & $\begin{array}{l}\text { Total } \\
\%(n)\end{array}$ \\
\hline Difficult eating & $46.4(32)$ & $14.1(13) * *$ & $28.0(35)$ & $45.0(27)$ & $25.0\left(30^{*}\right)$ & $31.7(57)$ & $29.6(102)$ \\
\hline Difficult speaking & $29.0(20)$ & $2.2(2)^{* *}$ & $13.7(22)$ & $30.0(18)$ & $14.2(17)^{*}$ & $19.4(35)$ & $16.5(57)$ \\
\hline Cleaning teeth & $14.5(10)$ & $4.3(4) *$ & $8.7(14)$ & $25.0(15)$ & $11.7(14)^{*}$ & $16.1(289)$ & $12.5(43)$ \\
\hline Sleeping & $36.2(25)$ & $6.5(6)$ & $19.3(31)$ & $25.0(15)$ & $14.2(17)$ & $17.8(32)$ & $18.3(63)$ \\
\hline Smiling & $15.9(11)$ & $3.3(3) *$ & $8.7(14)$ & $16.7(10)$ & $11.7(14)$ & $13.3(24)$ & $11.0(38)$ \\
\hline Emotional status & $21.7(15)$ & $1.1(1) * *$ & $9.9(16)$ & $10.0(6)$ & $7.5(9)$ & $8.3(15)$ & $9.0(31)$ \\
\hline Doing work & $14.5(10)$ & $1.1(1) *$ & $6.9(11)$ & $8.3(5)$ & $5.8(7)$ & $6.7(12)$ & $6.7(23)$ \\
\hline Enjoying contact & $10.1(7)$ & $1.1(1) *$ & $5.0(8)$ & $8.3(5)$ & $5.8(7)$ & $6.7(12)$ & $5.8(20)$ \\
\hline $\mathrm{OIDP}>0$ & $49.3(34)$ & $14.1(13)^{* * *}$ & $29.2(47)$ & $45(27)$ & $25.8(31)$ & $32.2(58)$ & $30.4(105)$ \\
\hline Cronbach's alpha & 0.90 & & 0.91 & 0.92 & & & \\
\hline
\end{tabular}

${ }^{*} p<0.10,{ }^{* *} p<0.05,{ }^{* * *} p<0.000$ 
variables in the multiple variable analysis, adjusted logistic regression analysis revealed that mother's perceived oral health status, caries experience, presence of gingival bleeding and total ECOHIS score associated significantly with OIDP status. The corresponding prevalence ratios (PR) and 95\% confidence intervals (CIs) were PR 0.3 (0.2-0.6); PR 1.8 (1.0-3.2); PR 1.1, (1.0-1.1); PR 2.1, (1.1-4.3), respectively. HIV status was not statistically significantly associated with mother's OIDP. Negative binomial regression revealed corresponding incidence rate ratios (IRR) and 95\% CI for for perceived oral health status were IRR 0.4 (0.2-0.7), caries experience IRR 1.8 (1.0-3.2), presence of gingival bleeding IRR1.1(1.0-1.2) and the total ECOHIS score IRR 2.3 (1.2-4.9). No statistically significant interaction between HIV status and socio-demographic and clinical covariates were observed indicating invariance of the socio-behavioural and clinical distribution of OIDP according to mothers' HIV status (Additional file 1).

\section{Discussion}

To our knowledge, this is the first study to estimate the prevalence as well as social- behavioural-, and clinical covariates of oral health related quality of life in Ugandan mothers living with HIV and their negative comparisons. The findings revealed that OHRQoL was substantially impaired in Ugandan mothers but did not discriminate between HIV positive and HIV negative participants. Neither was the socio-behavioural and clinical distribution of oral health related quality of life variant across HIV positive and negative mothers. Contrary to our expectations, the prevalence of oral impacts was about four percentage points higher in HIV negative than in HIV positive mothers regarding the total OIDP score and across the single OIDP items as well. The direction of this difference remained unchanged after adjusting for social-, behavioural-, and clinical covariates of OIDP that also varied between HIV positive- and negative mothers. The absence of significant difference in OIDP between

Table 3 Mothers' OIDP by HIV status, socio-demographic and self-reported clinical variables. Unadjusted and adjusted OLR and negative binomial regression

\begin{tabular}{|c|c|c|c|}
\hline & $\begin{array}{l}\text { OIDP }>0 \\
\%(n)\end{array}$ & $\begin{array}{l}\text { OIDP }>0 \\
\text { OR }(95 \% \mathrm{CI})\end{array}$ & $\begin{array}{l}\text { OIDP score range (0-8) } \\
\text { IRR }(95 \% \mathrm{Cl})\end{array}$ \\
\hline \multicolumn{4}{|l|}{ HIV status } \\
\hline Non infected & $32.0(58)$ & 1 & 1 \\
\hline Infected & $28.8(47)$ & $0.7(0.4-1.3)$ & $0.6(0.3-1.1)$ \\
\hline \multicolumn{4}{|l|}{ Age } \\
\hline $18-32$ & $31.5(50)$ & 1 & 1 \\
\hline $33+$ & $28.9(48)$ & $0.8(0.5-1.4)$ & $1.0(0.6-1.7)$ \\
\hline \multicolumn{4}{|l|}{ Education } \\
\hline End of primary & $30.4(56)$ & & \\
\hline Higher & $27.9(34)$ & & \\
\hline \multicolumn{4}{|l|}{ Oral health } \\
\hline Poor (very poor, poor, fair) & $47.3(61)$ & 1 & 1 \\
\hline Good (Good, very good) & $20.8(44) * * *$ & $0.3(0.2-0.6)$ & $0.4(0.2-0.7)$ \\
\hline \multicolumn{4}{|l|}{ Need for dental treatment } \\
\hline No & $23.8(71)$ & & \\
\hline Yes & $75.6(34) * * *$ & & \\
\hline \multicolumn{4}{|l|}{ Presence gingival bleeding } \\
\hline No & $25.6(41)$ & 1 & 1 \\
\hline Yes & $34.6(64) *$ & $1.8(1.0-3.2)$ & $1.8(1.0-3.2)$ \\
\hline \multicolumn{4}{|l|}{ Caries experience } \\
\hline No & $10.8(9)$ & 1 & 1 \\
\hline Yes & $36.6(96) * * *$ & $1.1(1.0-1.1)$ & $1.1(1.0-1.2)$ \\
\hline \multicolumn{4}{|l|}{ ECOHIS -total } \\
\hline No impact & $27.8(78)$ & 1 & 1 \\
\hline At least one impact & $41.4(24)^{* *}$ & $2.1(1.1-4.3)$ & $2.3(1.2-4.9)$ \\
\hline \multicolumn{4}{|l|}{ Information teeth received } \\
\hline No & $31.9(72)$ & & \\
\hline Yes & $26.7(31)$ & & \\
\hline
\end{tabular}

${ }^{*} p<0.10,{ }^{* *} p<0.05,{ }^{* * *} p<0.000$ 
the two groups of mothers investigated was probably not a consequence of negative confounding. In this study, OIDP items discriminated significantly according to mothers' self-reported oral health status indicating satisfactory conceptual validity across the HIV positive- and negative mothers investigated.

A strength of this study is the inclusion of a comparison group of HIV negative mothers which further strengthens the observed findings and is contrary to most previous studies lacking a separate control group. Another strength is the use of a validated OIDP scale previously reported to be appropriate in the Ugandan context [9, 33]. The main limitation of this the study may have been loss to follow-up from the original cohort of mothers living with HIV which might limit the interpretation of the findings as the HIV cohort may suffer from being underpowered. However as reported previously [34], the analysis comparing baseline characteristics of HIV positive mothers followed-up and those lost to follow-up showed similar distributions. In terms of generalisability, the mothers with HIV in this study may be different from the population of HIV positive mothers because they were recruited to participate in a trial. However, in terms of accessing the health care system, the mothers participating in this study might be representative of Uganda mothers with HIV as their contact with the health care system is similar.

Systematic reviews have found that pregnant women had poor knowledge and awareness of oral health [35, 36]. Bindu et al. [37] also found that pregnant women placed lower priority for oral and fear for dental services were reasons for delaying dental services. Also, higher parity has been associated with number teeth present, functional tooth units of natural teeth regardless of socio-demographic and behavioural variables [38, 39]. Women of lower parity in Nigeria tended to have better oral health [40]. This study did not assess factors related to pregnancy, parity, psychosocial related factors such as attitude knowledge, attitude, and behaviour thus the absence their assessment could have limited the findings. Future studies should therefore regard the mentioned factors.

Overall, the high prevalence of OIDP observed among the participants in this study, was lower than that reported earlier among Sudanese dental patients amounting to $33.3 \%$ and $18.7 \%$ among HIV positive and HIV negative participants from the general population having the same age distribution [41], but similar to the prevalence observed in the general population of pregnant women in Uganda (i.e. $25 \%$ and $30 \%$ in urban and rural areas) [10]. Other studies emanating from adult populations in industrialized and non-industrialized countries have reported prevalence rates of OIDP ranging from $18.3 \%$ to $75 \%$ in Norway and Malaysia, respectively [42-46].

As shown in Table 2, and in accordance with previous reports from sub-Saharan Africa [10, 47-49], physical aspects in terms of difficulty with eating, speaking and sleeping were the impacts most frequently reported in both groups of mothers investigated. A recent review of HIV patients showed, accordingly that the most affected aspects of oral health related quality of life were problems with eating followed by social disability in terms of difficulty doing usual job and being irritated that were less important [11]. In contrast, HIV positive people and individuals belonging to other marginalized groups such as drug abusers living in different cultural contexts have emphasized social impacts of their oral diseases as most disabling [13].

Independent of HIV status, mothers who perceived good oral health status were less likely whereas those who presented with gingival bleeding and dental caries experience were marginally more likely to report any oral impacts on daily performances (OIDP $>0$ ). This confirms the clinical gradient in oral health related quality of life that has been observed across HIV positive and negative populations worldwide $[10,11,14,50,51]$. Although, as shown by previous studies [11, 14], the disease burden in terms of the prevalence of gingival bleeding and caries experience were higher in HIV positive compared to negative mothers, this gradient was not reflected in their reported impacts on daily performances. This draws attention to the conceptual difference between clinicaland subjective indicators of oral health [8]. Subjective experience of oral health is influenced by cultural and social aspects not captured by the single clinical measurements of dental caries and periodontal disease. In accordance with the present finding, an Australian study revealed that HIV positive men were disadvantaged with regard to social impacts of their oral diseases, and that clinical indicators in terms of dental caries and periodontal disease were better among the HIV positive men than in the comparison group of general patients [12]. Similarities in oral health related quality of life among the two groups of mothers investigated in this study might be attributed to HIV positive mothers being in good health, presenting with high $\mathrm{CD} 4$ count which indicate optimal immunity. Also, as per treatment protocols, PLHIV on ART have routine two weekly review visits with health workers and this consultation with the health system may explain their better health quality of life compared to the control mothers who usually get in contact with health workers in response to symptoms. In addition, the fact that HIV positive mothers reported to be more frequently informed about oral health than their control counterparts might indicate appropriate access to 
health- and oral health care services. A previous study from industrialized country have shown that HIV positive women reported more frequent dental care utilization than did their HIV negative counterparts [14].

In this study mothers' OIDP- and the ECOHIS scores pertaining to children's and the family's perceived disadvantages due to child's oral diseases, associated significantly both in crude and adjusted analyses. This association might be viewed as a consequence of common risk factors for both OIDP and ECOHIS scores. Previous studies have shown that mother's caries experience- a risk factor for OIDP scores, associate with caries experience of their children, which in turn have negative impacts on children's and the family's quality of life [21]. According to Bandura's social cognitive theory, children's oral health outcomes are considered a modelling process whereby children imitate their parents' behaviours and recognize them as valued role models [52].

Most oral health promotion programmes in the Ugandan society are implemented at school level while curative services are conducted at health facility level and as outreach activities. Given the high OIDP documented in this study it is necessary to strengthen oral health prevention or promotion awareness beyond the schools and health facilities such as the media.

\section{Conclusion}

In conclusion, OHRQoL was substantially impaired in Ugandan mothers but did not discriminate between HIV positive and HIV negative participants. Mothers with impaired OHRQoL were more likely to have dental caries and children with impaired oral health related quality of life. HIV positive and negative mothers in Uganda deserve especial attention regarding their oral disease and quality of life status.

\footnotetext{
Abbreviations

ART: Antiretroviral therapy; Cl: Confidence interval; CPI: Community Periodontal impact scale; ECOHIS: Early childhood oral health impact scale; DMFT: Decayed missing filled teeth surfaces; HAART: Highly active antiretroviral therapy; HEU: HIV exposed uninfected; HIV: Human deficiency virus; HRQOL: Health related quality of life; HUU: HIV unexposed uninfected; IQR: Interquartile range; IRR: Incidence rate ratio; OHRQoL: Inter class correlation; OIDP: Ora health impacts on daily performance; NVP: Nevirapine; OR: Odds ratio; PLHIV: Persons living with Human Immune Virus; PMTCT: Prevention of mother to child transmission; PROMISE-PEP: Promoting infant health and nutrition in Sub-Saharan Africa: Safety and efficacy of infant Peri-Exposure Prophylaxis; PROMISE-PEP-M\&S: Promoting infant health and nutrition in Sub-Saharan Africa: Safety and efficacy of infant Peri-Exposure Prophylaxis to prevent HIV-1 transmission by breastfeeding-Mechanisms \& Safety; WHO: World Health Organisation; ZVD: Zidovudine; 3TC: Lamivudine.
}

\section{Supplementary Information}

The online version contains supplementary material available at https://doi. org/10.1186/s12955-021-01844-3.

Additional file 1. Table S1. Mothers' OIDP by HIV status, socio-demographic and self-reported clinical variables. Unadjusted and adjusted OLR and negative binomial regression. Table S2. Evaluation of effects of interaction of covariates* with HIV exposure regressed with oral health impacts.

\section{Acknowledgements}

This study used data collected in the context of the ANRS 12174 clinical trial and 12341 study. We are grateful to the ANRS 12174 and 12341 for sharing data and giving us the opportunity to carry out oral assessments. We acknowledge Dr. Matovu Mohammed (MM) for assisting with oral examinations.

\section{Authors' contributions}

NB, AN, IMSE JKT, LTF and SAL made contributions to conception and design of the study. NB, AN and JKT contributed to data acquisition. NB, AN, IMSE, JKT, LTF and SAL analysed, interpreted, drafted and gave final approval to this version of the manuscript. All authors read and approved the final manuscript.

\section{Author information}

NB is a dentist and is a postdoctoral fellow at the Department of Clinical Dentistry, University of Bergen, Norway. Årstadveien, 195009.

\section{Funding}

This study used data collected in the context of the ANRS 12174 clinical trial and 12341 study supported by the Pierre Bergé endowment fund in collaboration with SIDACTION and sponsored by the French National Agency for Research on AIDS and Viral Hepatitis (ANRS\#12341), the European Developing Countries Clinical Trials Partnership (EDCTP; grant number CT.2006.33020.004), the Research Council of Norway (GlobVac grant number 183600) and the Total Foundation. NB benefited from a postdoctoral research position funded by the Department of Clinical Dentistry, Faculty of Medicine, University of Bergen.

\section{Availability of data and materials}

The datasets used and/or analysed during the current study are available from the corresponding author on reasonable request.

\section{Declarations}

Ethics approval and consent to participate.

Ethical clearance was obtained from the School of Medicine Research and Ethics committee, Makerere University (SOMREC)-REC -030, Uganda National Council of Science and Technology (UNCST)-HS 2373 and the Regional Committee for Health and Medical Research (REK) —2017/760/REC sør-øst C from Norway.

\section{Consent for publication}

Not applicable.

\section{Competing interests}

The authors declare no competing interests.

\section{Author details}

${ }^{1}$ Department of Clinical Dentistry, University of Bergen, Bergen, Norway. ${ }^{2}$ Department of Global Public Health and Primary Care, University of Bergen, Bergen, Norway. ${ }^{3}$ Department of Addiction Medicine, Haukeland University Hospital, Bergen, Norway. ${ }^{4}$ Department of Global Public Health and Primary Care, Centre for International Health, University of Bergen, Bergen, Norway. 
${ }^{5}$ Department of Paediatrics' and Child Health, School of Medicine, College of Health Sciences, Makerere University, Kampala, Uganda.

Received: 5 February 2021 Accepted: 15 August 2021 Published online: 23 August 2021

\section{References}

1. UNAIDS. Global HIV \& AIDS statistics—2019 fact sheet: UNAIDS. 2019.

2. Kassebaum NJ, Smith AGC, Bernabé E, Fleming TD, Reynolds AE, Vos T, et al. Global, regional, and national prevalence, incidence, and disabilityadjusted life years for oral conditions for 195 countries, 1990-2015: a systematic analysis for the global burden of diseases, injuries, and risk factors. J Dent Res. 2017;96:380-7.

3. Cooper V, Clatworthy J, Harding R, Whetham J, Brown A, Leon A, et al. Measuring quality of life among people living with HIV: a systematic review of reviews. Health Qual Life Outcomes. 2017;15:220.

4. Sischo L, Broder HL. Oral health-related quality of life: what, why, how, and future implications. J Dent Res. 2011;90:1264-70.

5. Haag DG, Peres KG, Balasubramanian M, Brennan DS. Oral conditions and health-related quality of life: a systematic review. J Dent Res. 2017;96:864-74.

6. Adulyanon S, Vourapukjaru J, Sheiham A. Oral impacts affecting daily performance in a low dental disease Thai population. Community Dent Oral Epidemiol. 1996;24:385-9.

7. Badley EM. The ICIDH: format, application in different settings, and distinction between disability and handicap. A critique of papers on the application of the International Classification of Impairments, Disabilities, and Handicaps. Int Disabil Stud. 1987;9:122-5.

8. Locker D. Measuring oral health: a conceptual framework. Community Dent Health. 1988;5:3-18.

9. Astrom AN, Okullo I. Validity and reliability of the Oral Impacts on Daily Performance (OIDP) frequency scale: a cross-sectional study of adolescents in Uganda. BMC Oral Health. 2003:3:5.

10. Wandera MN, Engebretsen IM, Rwenyonyi CM, Tumwine J, Astrom AN, Group P-ES. Periodontal status, tooth loss and self-reported periodontal problems effects on oral impacts on daily performances, OIDP, in pregnant women in Uganda: a cross-sectional study. Health Qual Life Outcomes. 2009;7:89.

11. Muralidharan S, Mahendrakar S, Talekar A, Nara A, Kanitkar AA, Kanitkar A, et al. Oral health-related quality of life in hiv: a systematic review. J Contemp Dent Pract. 2020;21:585-92.

12. Coates E, Slade GD, Goss AN, Gorkic E. Oral conditions and their social impact among HIV dental patients. Aust Dent J. 1996;41:33-6.

13. Liberali SA, Coates EA, Freeman AD, Logan RM, Jamieson L, Mejia G. Oral conditions and their social impact among HIV dental patients, 18 years on. Aust Dent J. 2013;58:18-25.

14. Mulligan R, Seirawan H, Alves ME, Navazesh M, Phelan JA, Greenspan D, et al. Oral health-related quality of life among HIV-infected and at-risk women. Community Dent Oral Epidemiol. 2008;36:549-57.

15. Rocha Trindade R, Marques J, Veiga M, Marques D, Mata A. HIV-1 impact on oral health-related quality of life: a cross-sectional study. AIDS Care. 2020:1-8.

16. Tomar SL, Pereyra M, Metsch LR. Oral health-related quality of life among low-income adults living with HIV. J Public Health Dent. 2011;71:241-7.

17. Parish CL, Feaster DJ, Pereyra MR, Alcaide ML, Weber KM, Cohen M, et al. Oral health-related quality of life and unmet dental needs among women living with HIV. J Am Dent Assoc. 2020;151:527-35.

18. da Costa W, Lins L, Sarmento VA, Netto EM, Brites C. Oral health and health-related quality of life in HIV patients. BMC Oral Health. 2018;18(1):151.

19. Soares GB, Garbin CA, Rovida TA, Garbin AJ. Oral health associated with quality of life of people living with HIV/AIDS in Brazil. Health Qual Life Outcomes. 2014;12:28.

20. de Quadros Coelho M, Cordeiro JM, Vargas AM, de Barros Lima Martins AM, de Almeida Santa Rosa TT, Senna Ml, et al. Functional and psychosocial impact of oral disorders and quality of life of people living with HIV/ AIDS. Qual Life Res. 2015;24:503-11.

21. Birungi N, Fadnes LT, Nankabirwa V, Tumwine JK, Astrom AN. Caretaker's caries experience and its association with early childhood caries and children's oral health-related quality of life: a prospective two-generation study. Acta Odontol Scand. 2016;74:605-12.

22. Weintraub J, Prakash P, Shain S, Laccabue M, Gansky S. Mothers' caries increases odds of children's caries. J Dent Res. 2010;89:954-8.

23. Wigen TI, Wang NJ. Maternal health and lifestyle, and caries experience in preschool children. A longitudinal study from pregnancy to age $5 \mathrm{yr}$. Eur $\mathrm{J}$ Oral Sci. 2011;119:463-8.

24. Massarente DB, Domaneschi C, Marques HH, Andrade SB, Goursand D, Antunes JL. Oral health-related quality of life of paediatric patients with AIDS. BMC Oral Health. 2011;1:2.

25. Rovaris NS, Galato D, Schuelter-Trevisol F, da Silva J, Linhar Lda S, Nickel DA, et al. Oral health status and its impact on the quality of life of children and adolescents living with HIV-1. BMC Res Notes. 2014;7:478.

26. Jeganathan S, Carey H, Purnomo J. Impact of xerostomia on oral health and quality of life among adults infected with HIV-1. Spec Care Dentist. 2012;32:130-5.

27. Nagot N, Kankasa C, Tumwine JK, Meda N, Hofmeyr GJ, Vallo R, et al. Extended pre-exposure prophylaxis with lopinavir-ritonavir versus lamivudine to prevent HIV-1 transmission through breastfeeding up to 50 weeks in infants in Africa (ANRS 12174): a randomised controlled trial. Lancet. 2016;387:566-73.

28. Galiwango RM, Musoke R, Lubyayi L, Ssekubugu R, Kalibbala S, Ssekweyama V, et al. Evaluation of current rapid HIV test algorithms in Rakai. Uganda J Virol Methods. 2013;192:25-7.

29. Birungi N, Fadnes LT, Okullo I, Kasangaki A, Nankabirwa V, Ndeezi G, et al. Effect of breastfeeding promotion on early childhood caries and breastfeeding duration among 5 year old children in Eastern Uganda: a cluster randomized trial. PLoS ONE. 2015;10:e0125352.

30. Masumo R, Bardsen A, Mashoto K, Astrom AN. Child- and family impacts of infants' oral conditions in Tanzania and Uganda- a cross sectional study. BMC Oral Health. 2012;5:538.

31. Bernabé E, Tsakos G, Sheiham A. Intensity and extent of oral impacts on daily performances by type of self-perceived oral problems. Eur J Oral Sci. 2007;115:111-6.

32. Who. Oral Health Surveys: Basic Methods (5th Edition). Albany, NY, USA: World Health Organization; 2013.

33. Wandera M, Engebretsen IM, Okullo I, Tumwine JK, Astrom AN, Group P-ES. Socio-demographic factors related to periodontal status and tooth loss of pregnant women in Mbale district, Uganda. BMC Oral Health. 2009;9:18.

34. Birungi N, Fadnes LT, Engebretsen IMS, Lie SA, Tumwine JK, Åstrøm AN, et al. Caries experience and oral health related quality of life in a cohort of Ugandan HIV-1 exposed uninfected children compared with a matched cohort of HIV unexposed uninfected children. BMC Public Health. 2020;20:423.

35. Gambhir RS, Nirola A, Gupta T, Sekhon TS, Anand S. Oral health knowledge and awareness among pregnant women in India: a systematic review. J Indian Soc Periodontol. 2015;19(6):612.

36. Fakheran O, Saied-Moallemi Z, Khademi A, Sahebkar A. Oral healthrelated quality of life during pregnancy: a systematic review. Curr Pharm Des. 2020;26(32):4014-21.

37. Bhaskar B, Thomas S, Kumar J, Gomez M. Self-perception on oral health and related behaviours among antenatal mothers attending a public antenatal clinic_Kerala. J Family Med Prim Care. 2020;9:4396-400.

38. Ueno M, Ohara S, Inoue M, Tsugane S, Kawaguchi Y. Association between parity and dentition status among Japanese women: Japan public health center-based oral health study. BMC Public Health. 2013;13:1-6.

39. Cozier YC, Heaton B, Bethea TN, Freudenheim JL, Garcia RI, Rosenberg L. Predictors of self-reported oral health in the Black Women's Health Study. J Public Health Dent. 2020;80:70-8.

40. Bukar M, Audu BM, Adesina OA, Marupa JY. Oral health practices among pregnant women in North Eastern Nigeria. Niger J Clin Pract. 2012;15:302-5.

41. Nasir EF, Marthinussen MC, Åstrøm AN. HIV/AIDS-related attitudes and oral impacts on daily performances: a cross-sectional study of Sudanese adult dental patients. BMC Health Serv Res. 2013;13:335.

42. Åstrøm A, Haugejorden O, Skaret E, TrovikT, Klock K. Oral impacts on daily performance in Norwegian adults: the influence of age, number of missing teeth, and socio-demographic factors. Eur J Oral Sci. 2006;114:115-21. 
43. Zeng X, Sheiham A, Bernabé E, Tsakos G. Relationship between dental status and Oral Impacts on Daily Performances in older Southern Chinese people. J Public Health Dent. 2010;70:101-7.

44. Kida IA, Åstrøm AN, Strand GV, Masalu JR, Tsakos G. Psychometric properties and the prevalence, intensity and causes of oral impacts on daily performance (OIDP) in a population of older Tanzanians. Health Qual life Outcomes. 2006:4:56

45. Erić J, Stančić I, Šojić LT, Popovac AJ, Tsakos G. Validity and reliability of the oral impacts on daily performance (OIDP) scale in the elderly population of Bosnia and Herzegovina. Gerodontology. 2012;29:e902-8.

46. Ostberg A-L, Andersson P, Hakeberg M. Cross-cultural adaptation and validation of the oral limpacts on daily performances (OIDP) in Swedish. Swed Dent J. 2008;32:187-95.

47. Mbawalla HS, Masalu JR, Åstrøm AN. Socio-demographic and behavioural correlates of oral hygiene status and oral health related quality of life, the Limpopo-Arusha school health project (LASH): a cross-sectional study. BMC Pediatr. 2010;10:87

48. Mashoto KO, Åstrøm AN, Skeie MS, Masalu JR. Changes in the quality of life of Tanzanian school children after treatment interventions using the Child-OIDP. Eur J Oral Sci. 2010;118:626-34.
49. Simangwa LD, Johansson A-K, Johansson A, Minja IK, Åstrøm AN. Oral impacts on daily performances and its socio-demographic and clinical distribution: a cross-sectional study of adolescents living in Maasai population areas, Tanzania. Health Qual Life Outcomes. 2020;18:1-181.

50. Nasir EF, Åstrøm AN, David J, Ali RW. Utilization of dental health care services in context of the HIV epidemic-a cross-sectional study of dental patients in the Sudan. BMC Oral Health. 2009;9:30.

51. Tsakos G, Steele JG, Marcenes W, Walls AW, Sheiham A. Clinical correlates of oral health-related quality of life: evidence from a national sample of British older people. Eur J Oral Sci. 2006;114:391-5.

52. Bandura A. Social cognitive theory: an agentic perspective. Annu Rev Psychol. 2001;52:1-26.

\section{Publisher's Note}

Springer Nature remains neutral with regard to jurisdictional claims in published maps and institutional affiliations.
Ready to submit your research? Choose BMC and benefit from:

- fast, convenient online submission

- thorough peer review by experienced researchers in your field

- rapid publication on acceptance

- support for research data, including large and complex data types

- gold Open Access which fosters wider collaboration and increased citations

- maximum visibility for your research: over $100 \mathrm{M}$ website views per year

At BMC, research is always in progress.

Learn more biomedcentral.com/submissions 